\title{
11 Physical activity level
}

For reasons of uniformity, it has become common international practice to represent the average daily energy requirement for physical activity as a proportion of the basal metabolic rate. Accordingly, this measure of metabolic energy requirements is called the "physical activity level" (PAL).

\section{$\mathrm{PAL}=$ total energy expenditure/ \\ basal metabolic rate (TEE/BMR)}

The advantage of this approach is that certain factors influencing energy requirements such as age, gender and body weight are already factored into the equation, thus enabling us to compare the energy expenditure for defined physical activities in different types of people. The daily energy expend- iture is estimated by multiplying the duration of the individual activities by the value for the respective basal metabolic rate (Yamada et al. 2013, Westerterp 2013).

As an example, a 24-hour period comprising 8 hours of work with a high energy requirement of 2.4 PAL, 8 hours of work with an average energy expenditure of 1.6 PAL and 8 hours of sleep with $0.95 \mathrm{PAL}$, adds up to a PAL of

$$
(2.4 \times 8+1.6 \times 8+0.95 \times 8): 24=\mathbf{1 . 6 5}
$$

In persons engaging in athletic activities totaling 3-5 hours per week, 0.3 PAL units per day can be added to the respectively calculated values. -Tab. 11.1 lists some of the PAL values describing common activities as formulated by the German Nutrition Society.

- Tab. 11.1 Energy expenditure for various activities measured by basal metabolic rate

\begin{tabular}{|l|l|l|}
\hline Type of physical activity & PAL & Examples \\
\hline Heavy occupational work & $2.0-2.4$ & $\begin{array}{l}\text { Construction workers, farmers, high perfor- } \\
\text { mance athletes }\end{array}$ \\
\hline Predominantly standing or walking work & $1.8-1.9$ & $\begin{array}{l}\text { Homemakers, salespersons, restaurant servers, } \\
\text { mechanics, traders }\end{array}$ \\
\hline $\begin{array}{l}\text { Sedentary activity/seated work with some requirement } \\
\text { for occasional walking or standing work }\end{array}$ & $1.6-1.7$ & Drivers, laboratory assistants, students \\
\hline seated work with little or no strenuous leisure activity & $1.4-1.5$ & Office employees, precision mechanics \\
\hline Exclusively sedentary or bedridden lifestyle & 1.2 & Old, infirm individuals \\
\hline Sleep & 0.95 & \\
\hline
\end{tabular}

PAL Physical Activity Level 